Document downloaded from:

http://hdl.handle.net/10251/178910

This paper must be cited as:

Gil Pascual, M.; Pastor-Ricós, F.; Torres Bosch, MV.; Vos, TE. (2020). DECODER -

DEveloper COmpanion for Documented and annotatEd code Reference. Springer. 643-644. http://hdl.handle.net/10251/178910

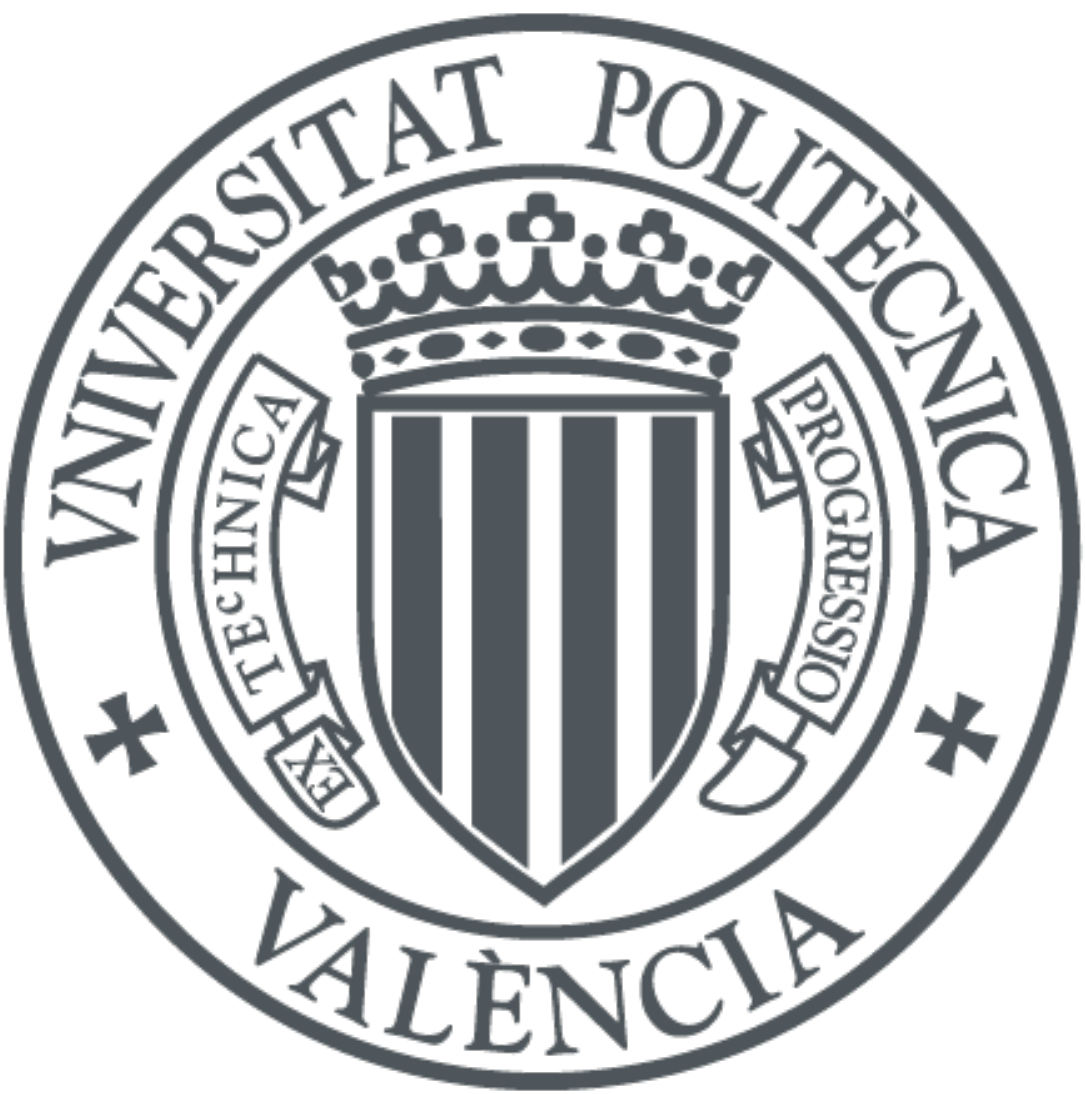

The final publication is available at

https://doi.org/10.1007/978-3-030-50316-1_1

Copyright Springer

Additional Information 


\title{
DECODER - DEveloper COmpanion for Documented and annotatEd code Reference ${ }^{\star}$
}

\author{
Miriam Gil, Fernando Pastor Ricos, Victoria Torres, and Tanja E.J. Vos ${ }^{\natural}$ \\ Universitat Politècnica de València \\ \{mgil,fpastor, vtorres, tvos\}@pros.upv.es \\ www.decoder-project.eu (2019-2022)
}

\section{Summary of the project}

\section{$1.1 \quad$ Objectives}

The influence of software on our daily lives is continuously growing. Software is everywhere. Unfortunately, high quality software is not everywhere. Studies show that software failures exist and they have far-reaching implications in terms of money, safety and privacy. Hence, guaranteeing the quality of software throughout all the different development processes is increasingly important. However, in practice many things often go wrong during software development projects due to uninformed decisions being taken along the whole process. And these are not isolated cases, some already go as far as arguing that there are signs of a 'coming software apocalypse' [1]. Reasons for taking the wrong decisions during software development are: (1) the amount of information, and (2) the lack of proper documentation.

During a typical development process there are interactions of many stakeholders, at very different abstraction levels, and often over ambiguous and incomplete documents. This makes the integration and even more the maintenance of software systems extremely difficult and costly. The DECODER project proposes a solution to this problem having as its main objective:

Build a smart environment that could assist and help developers, analysts, reviewers and testers to improve the software development process.

The DECODER environment will give support to properly handle project knowledge derived from all different software development artefacts like: source code, specifications, informal documents, tests, etc.. DECODER will provide a map through the software project intelligence fulfilling the need for instantaneous access to its documentation, abstract models, verification data and traceability matrix. This is needed to take better decisions.

\footnotetext{
* This work has been developed with the financial support of the European Union's
} Horizon 2020 research and innovation programme under grant agreement No. 824231 


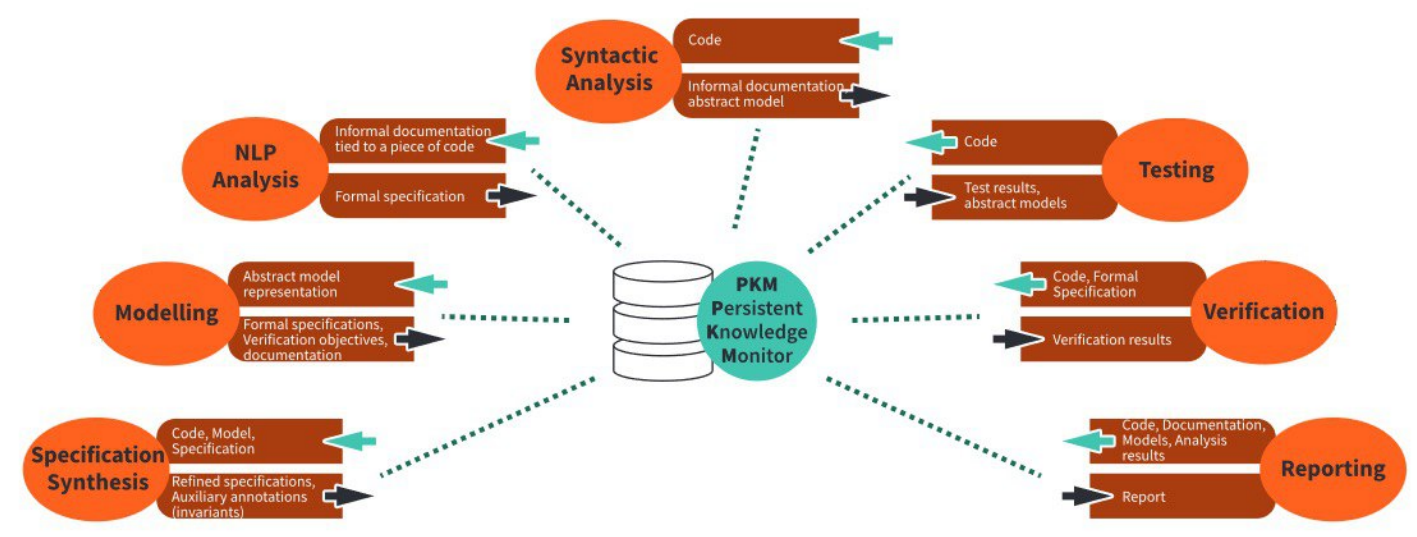

Fig. 1. The Persistent Knowledge Monitor and its surrounding tools

\section{$1.2 \quad$ Expected tangible result}

One of the core results of DECODER is the Persistent Knowledge Monitor (PKM). It will provide a "central" infrastructure to store, access, and trace all the persistent data, information and knowledge related to a given software or ecosystem. The rest of the project results are: tools for feeding the PKM with artefacts from different phases of the development lifecycle, tools for querying the PKM to obtain information to make the right decisions during different phases of the development lifecycle. The PKM will have a common communication schema to facilitate these interactions. This is depicted in Figure 1.

\section{Summary of current project results}

At the current status of the project, there is a first release of the PKM as a MongoDB together with a PKM API for queries, creating reports and management of users, access to artefacts or the traceability matrix. At the same time the tools for feeding and querying are being connected and different techniques are being researched for generating knowledge (see Figure 1). A methodology has been defined for different stakeholders on how the PKM can be used to assist them in their responsibilities along the software development life-cycle. Stakeholders that are distinguished are: developers, reviewers, and maintainers. Their tasks, and the techniques they use, have been studied and the different artefacts have been mapped onto these. Finally, the pilot systems have been defined and research questions have been defined for the case studies that will evaluate the DECODER results.

\section{References}

1. Somers, J.: The coming software apocalypse. The Atlantic 26 (2017) 